\title{
Endoscopic ultrasound in the monitoring of the intestinal allograft
}

\author{
Mihai Oltean, ${ }^{1,2}$ Per Hedenström (10 , ${ }^{3,4}$ Jonas Varkey, ${ }^{3,4}$ Gustaf Herlenius, ${ }^{1,2}$ \\ Riadh Sadik ${ }^{3,4}$
}

To cite: Oltean $M$

Hedenström P, Varkey J, et al. Endoscopic ultrasound in the monitoring of the intestinal allograft. BMJ Open Gastro 2022:9:e000792. doi:10.1136/ bmjgast-2021-000792

$\mathrm{MO}$ and $\mathrm{PH}$ are joint first authors.

Received 22 September 2021 Accepted 10 January 2022

Check for updates

(c) Author(s) (or their employer(s)) 2022. Re-use permitted under CC BY-NC. No commercial re-use. See rights and permissions. Published by BMJ.

${ }^{1}$ Department of Surgery, Institute for Clinical Sciences, University of Gothenburg, Gothenburg, Sweden

${ }^{2}$ Transplant Institute, Sahlgrenska University Hospital, Gothenburg, Sweden ${ }^{3}$ Department of Molecular and Clinical Medicine, Institute of Medicine, University of Gothenburg, Gothenburg, Sweden

${ }^{4}$ Division of Gastroenterology, Department of Medicine, Sahlgrenska University Hospital, Gothenburg, Sweden

Correspondence to Dr Mihai Oltean; mihai.oltean@surgery.gu.se

\begin{abstract}
Objective Chronic rejection (CR) of the small intestinal allograft includes mucosal fibrosis, bowel thickening and arteriopathy in the outer wall layers and the mesentery. CR lacks non-invasive markers and reliable diagnostic methods. We evaluated endoscopic ultrasound (EUS) as a novel approach for monitoring of the intestinal allograft with respect to $\mathrm{CR}$.
\end{abstract}

Design In intestinal graft recipients, EUS and enteroscopy with ileal mucosal biopsy were performed via the ileostomy. At EUS, the wall thickness of the intestinal graft was measured in standard mode, whereas the resistive index (RI) of the supplying artery was assessed in pulsed Doppler mode. At enteroscopy, the intestinal mucosa was assessed. Findings were compared with histopathology and clinical follow-up.

Results EUS was successfully performed in all 11 patients (adequate clinical course (AC) $n=9 ; C R n=2$ ) after a median interval of 1537 days (range: 170-5204), post-transplantation. The total diameter of the wall (layer I-V) was comparable in all patients. Meanwhile, the diameter of the outermost part (layer IV-V; that is, muscularis propria-serosa) was among the two CR patients (range: 1.3-1.4 mm) in the upper end of measurements as compared with the nine AC patients (range: $0.5-1.4 \mathrm{~mm}$ ). The RI was $>0.9$ in both CR patients, while the RI was $\leq 0.8$ in all AC patients. Both CR patients had abnormal findings at enteroscopy and histopathology and deceased during follow-up.

Conclusion EUS is a promising tool providing detailed information on the intestinal graft morphology and rheology, which may be used for assessment of potential CR in long-term follow-up of intestinal allograft recipients.

\section{BACKGROUND}

Intestinal transplantation (ITx), either alone or in combination with other splanchnic organs, is a relatively rare and challenging type of organ transplantation. The early results of ITx have continuously improved over the last decades with 1-year patient and graft survival increasing from less than $60 \%$ to over $80 \% .^{1{ }^{2}}$ However, long-term results have remained unchanged with 5-year and 10-year graft survival of around $50 \% .^{3}{ }^{4} \mathrm{~A}$ major cause for graft attrition is chronic rejection (CR), a puzzling clinical and pathologic condition ascribed to both allogenic and alloindependent factors. Animal models suggest
Summary box

What is already known about this subject?

- Chronic rejection (CR) of the small intestinal allograft is a potentially life-threatening condition, which is difficult to diagnose with non-invasive markers and routine imaging. Endoscopic ultrasound (EUS) can provide detailed images of the intestinal wall including supplying arteries. Therefore, EUS may contribute with important diagnostic information in suspected CR.

What are the new findings?

- We evaluated EUS, including measurement of the wall thickness and the resistive index (RI), for monitoring of the intestinal allograft with respect to $\mathrm{CR}$ in 11 intestinal graft recipients also subjected to enteroscopy with forceps biopsy. EUS was successfully performed in all patients-nine patients with adequate clinical course $(\mathrm{AC})$ and two patients with suspected CR. The total wall thickness was comparable in CR patients and AC patients. Meanwhile, the RI was recorded higher in both patients with $\mathrm{CR}$ as compared with AC patients. Both patients with CR had abnormal findings also at enteroscopy and histopathology.

How might it impact on clinical practice in the foreseeable future?

- The use of EUS in the monitoring of the intestinal allograft will be beneficial to gastroenterologists by providing diagnostic information on the risk for $\mathrm{CR}$, which can be challenging to obtain by other methods. Moreover, EUS is patient-friendly since it is a non-radiating type of imaging, which can be performed in an outpatient setting without any need for general anaesthesia.

that CR starts with mesenterial fibrosis and arteriolitis and later progresses with submucosal fibrosis. ${ }^{5}$ Unfortunately, all these earlier features occur outside the reach of endoscopic mucosal biopsies, which is the main method for intestinal graft surveillance. It is only in late, advanced stages that CR results in mucosal alterations and a clinical picture featuring motility impairment, bacterial overgrowth, enterocutaneous fistulas or weight loss, all suggestive of this ominous condition. 
Even so, the diagnosis of CR is usually confirmed only in full-thickness biopsies after removing the failed grafts. ${ }^{6}$

Ultrasound is an imaging technique whose noninvasiveness, rapid availability and low costs have propelled it as essential tool in transplantation medicine for the assessment of parenchymatous organs and fluid collections. $^{7}$ The growing expertise of examiners has expanded the use of intestinal ultrasound in the assessment of the gastrointestinal tract. Intestinal ultrasound has been shown to have high sensitivity and specificity as well as high positive and negative predictive value, in the detection or exclusion of intestinal inflammatory activity in inflammatory bowel disease (IBD) ${ }^{8-10}$ Moreover, the advent of endoscopic ultrasound (EUS) equipment has offered new possibilities for detailed, close range examination of structures laying inside or in close proximity of the gastrointestinal wall. ${ }^{11-13}$ EUS can accurately discriminate wall thickening, intraparietal circulation or inflammation. The equipment for EUS is fitted on regular endoscopes and allows both regular endoscopies and biopsies as well as a detailed assessment of intestinal wall and the neighbouring structures. With EUS, the intestinal wall appears as a five-layered structure: the mucosa (layer I, innermost, hyperechoic), the muscularis mucosae (layer II, hypoechoic), the submucosa (layer III, hyperechoic), the muscularis propria (layer IV, hypoechoic) and subserosa (layer $\mathrm{V}$, hyperechoic), figure 1 .

To date, the diagnosis of CR is delayed and the clinical decisions stemming from it such as graft removal or retransplantation are mostly based on clinical criteria, without having a well-defined marker or specific investigation. ${ }^{5}{ }^{12}$ Given the proven ability of intestinal EUS to assess bowel wall thickness, wall layer stratification as well as blood flow inside and close to the intestinal wall, we set up to assess the findings and diagnostic value of intestinal EUS in the long-term graft surveillance after ITx.

\section{METHODS}

\section{Patients and preparations}

All transplants were performed at Sahlgrenska University Hospital between 2007 and 2021. Patient characteristics are detailed in table 1. EUS and enteroscopy were performed in 11 intestinal graft recipients at variable intervals after transplantation at the time of scheduled, protocol endoscopic follow-up. No routine, cross-sectional imaging was performed during follow-up.

Two out of 11 patients had clinical suspicion of CR due to need for late reintroduction of parenteral nutrition, fistulae, recurrent sepsis episodes and protracted, unexplained abdominal pain in the absence of any other cause. These two patients died during the follow-up time frame, partly due to CR, which is further discussed below.

The remaining nine patients had stable graft function and adequate clinical course (AC). None of the patients had Crohns' disease.

All study participants were scheduled for a $90 \mathrm{~min}$ examination $(60 \mathrm{~min}$ for the procedure as such and $30 \mathrm{~min}$ for

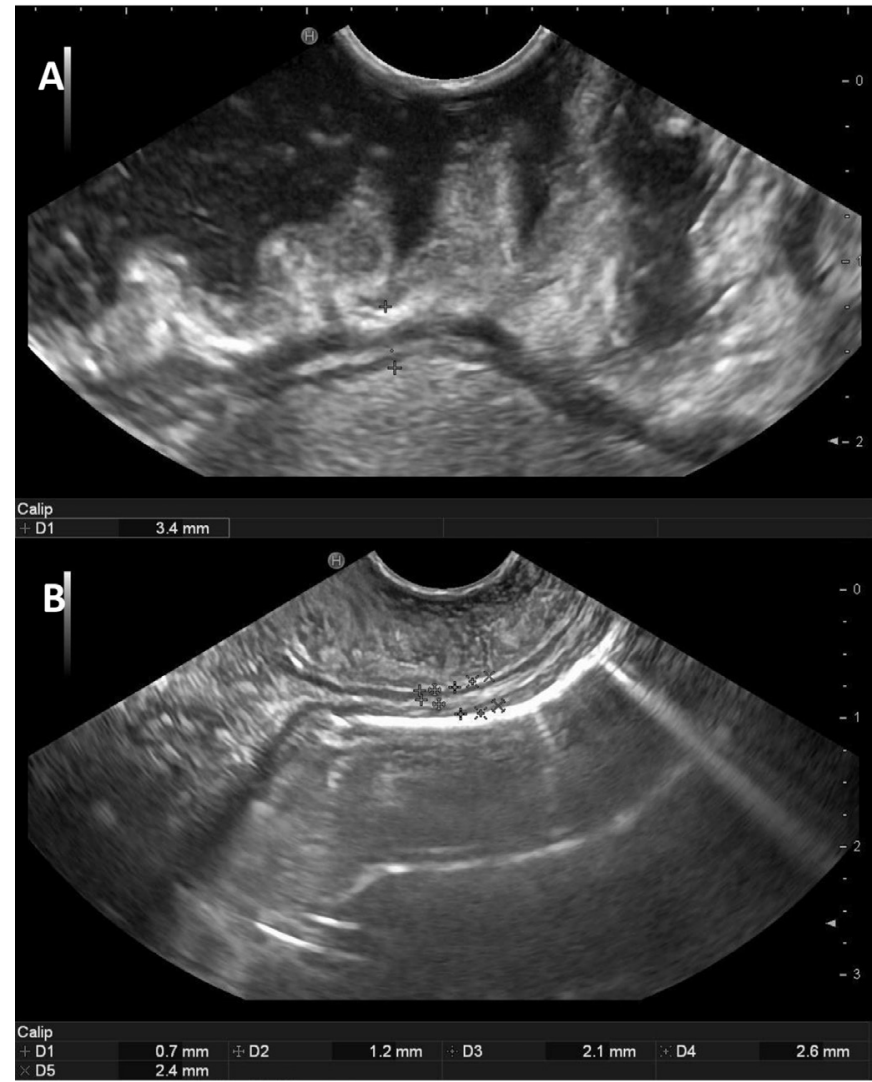

Figure 1 A: The measurement of the total thickness of the small bowel wall with the lumen appearing upwards. The EUS image shows the five wall layers of the small bowel presented as follows - layer I, innermost: mucosa (hyperechoic); layer II: muscularis mucosae (hypoechoic); layer III: submucosa (hyperechoic); layer IV: muscularis propria (hypoechoic); layer V, outermost: serosa (hyperechoic). The measurement of the complete bowel thickness (wall layer I-V) was performed by marking the distance between the first (innermost cross) and the fifth wall layer (outermost cross) at bowel relaxation. B: The measurement of innermost part of the bowel wall (wall layer I-III) was performed by marking the distance between the first and the third wall layer at bowel relaxation (D2 $-1.2 \mathrm{~mm}$, B). The diameter of the outermost part of the bowel wall was performed by subtracting the diameter of the innermost part from total wall diameter. EUS, endoscopic ultrasound.

postprocedural monitoring) at the endoscopy unit. The examinations were performed through the ileostomy without any bowel preparation apart from.

The patients were not examined under general anaesthesia, but sedation with intravenous midazolam and/or alphentanil was administered if requested by any of the patients. The study was approved by the Regional Ethical Review Board (Dnr 573-09).

\section{Equipment and procedures \\ Endoscopic ultrasound}

The procedures were performed by the study endosonographer $(\mathrm{PH})$ using an ultrasound processor (HI VISON Ascendus, Hitachi, Tokyo, Japan) and a linear echoendoscope (eg, 3870UTK, Pentax, Tokyo, Japan). In four 


\begin{tabular}{|c|c|c|c|c|c|c|}
\hline Patient & Sex & Age at transplant & Age at first EUS & Pre-transplant diagnosis & Graft type & Clinical course \\
\hline 1 & M & 54 & 56 & PMVT & MVTx & Adequate \\
\hline 2 & $\mathrm{~F}$ & 39 & 41 & SBS & MVTx & Adequate \\
\hline 3 & $\mathrm{~F}$ & 4 & 14 & SBS & ISB & Chronic rejection \\
\hline 4 & $\mathrm{~F}$ & 37 & 37 & SBS & MVTx & Adequate \\
\hline 5 & $\mathrm{~F}$ & 13 & 22 & SBS & ISB & Chronic rejection \\
\hline 6 & M & 40 & 45 & SBS & MVTx & Adequate \\
\hline 7 & M & 48 & 58 & SBS, PMVT & MVTx & Adequate \\
\hline 8 & M & 33 & 34 & SBS & MVTx & Adequate \\
\hline 9 & $\mathrm{~F}$ & 20 & 35 & CIPO & MVTx & Adequate \\
\hline 10 & $\mathrm{~F}$ & 46 & 46 & SBS & MVTx & Adequate \\
\hline 11 & $\mathrm{~F}$ & 12 & 19 & $\mathrm{AL}$ & MVTx & Adequate \\
\hline
\end{tabular}

AL, autoimmune leiomyositis; CIPO, chronic intestinal pseudoobstruction; EUS, endoscopic ultrasound; ISB, isolated small bowel overnight fasting; MVTx, multivisceral transplantation; PMVT, portomesenteric venous thrombosis; SBS, short bowel syndrome.

of the procedures, a second endosonographer (RS) was also present on-site. Without any insufflation of air, the echoendoscope was introduced $10-20 \mathrm{~cm}$ into the small bowel graft via the ileostomy. A small amount of lukewarm tap water was injected into the bowel lumen. Then, a cross-sectional ultrasound image of the bowel segment was centred on the screen during intestinal relaxation and the image was frozen. The thickness of the entire bowel wall (wall layer I-V), figure 1A, the innermost part of the bowel wall (wall layer I-III), and the outermost part of the bowel wall (wall layer IV-V) were measured and recorded. This procedure was repeated for at least three times at different locations. Finally, the median value of the three measurements was recorded and used for subsequent comparisons.

After bowel wall measurements, a mesenteric artery close to the bowel wall was identified by the use of colour Doppler, figure 2. Then, settings were changed to the pulsed wave mode and the resistive index (RI) was measured in the selected artery a previously described formula $^{14}$ : RI=(PSV-EDV)/PSV (PSV: peak systolic velocity; EDV: end diastolic velocity). In total, three RI-measurements were performed in each examination, and the median value of the three measurements was recorded and used for subsequent comparisons. For reasons related to the ultrasound software, the procedure of RI measurement was initiated after the start of the study and performed in nine patients during one EUS examination only.

\section{Enteroscopy}

Thereafter, a high-definition gastroscope (eg, 760Z, Fujifilm, Tokyo, Japan) was used for graft enteroscopy. The gastroscope was advanced $10-20 \mathrm{~cm}$ into the small bowel via the ileostomy. Then, the small bowel mucosa was carefully assessed in white light mode while capturing still images and video sequences. Assessment of the mucosa was performed both in standard view and close-up view and evaluated according to a previously described endoscopic score, ${ }^{15}$ figure 3 . In brief, G0 describes a normal mucosa with long, slender villi and no erythema, G1 stands for mild erythema and mucosal friability and slightly edematous villi, G2 represents a mucosa with marked erythema and mucosal friability, erosions, blunted villi, G3 entails spontaneous bleeding, ulcerations with effaced or lost villi, whereas G4 denotes extensive mucosal loss and visible submucosa. Finally, 3-5 mucosal random biopsies were collected using standard biopsy forceps.

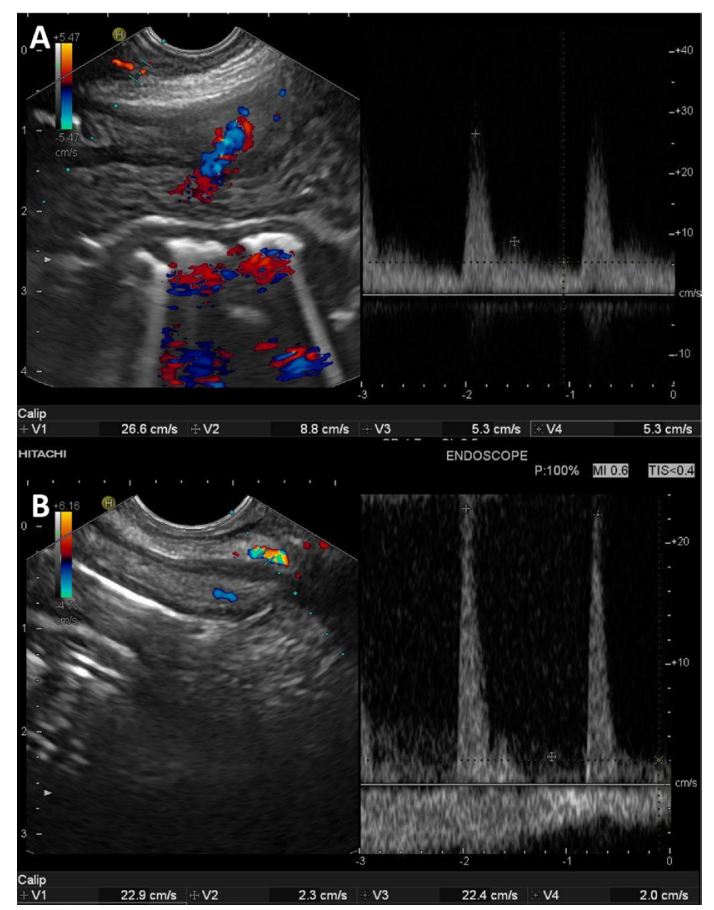

Figure 2 The measurement of the resistive index (RI). The endoscopic ultrasound (EUS) image of the small bowel in colour Doppler mode (CW) (left) and in pulsed Doppler mode (PW) (right) in a patient with an uncomplicated clinical course $(A)$ and in a patient with chronic rejection. The $R$ was calculated by dividing the end diastolic velocity (V2 and V4) with the peak systolic velocity (V1 and V4). 


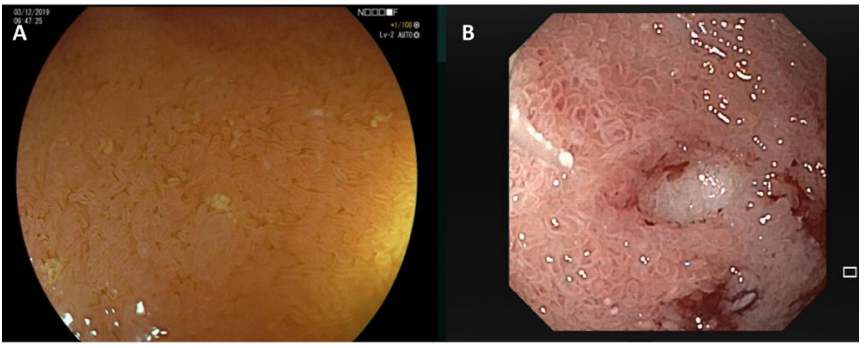

Figure 3 Enteroscopy of an intestinal graft with adequate clinical course (A) and chronic rejection (B).

\section{Histopathology}

The mucosal biopsies were assessed by an experienced transplant pathologist according to established criteria. ${ }^{16}$

Intestinal segments were obtained at the time of ileostomy removal in two patients with uneventful course (patient 2 and 6 after 420 and 1962 days posttransplantation, respectively) and in patient 3 at the time of intestinal graft removal due to CR (4890 days posttransplantation). All three full-thickness biopsies were obtained within a month from the EUS examination. These samples were used to compare the EUS measurements with full thickness intestinal biopsies.

\section{Study outcomes}

The coprimary study outcomes were the feasibility and the accuracy of measurements at EUS in identifying patients with and without clinical suspicion of CR in the intestinal graft.

\section{Statistics}

The dispersion of the recorded data was described as median and range. Given the limited number of patients included in the study, numerical comparisons of data were performed between the group of patients with AC and the patients with CR. However, no statistical comparisons with $\mathrm{p}$ value calculation were performed. For the same reason, no $\mathrm{p}$ value analysis was performed regarding the RI measurement. Data analysis was performed using IBM SPSS Statistics V.25.0.

\section{RESULTS}

Eight out of 11 patients (patient 1 to 8 ) underwent two EUS examinations, whereas the remaining three patients (patient 9 to 11) underwent only one EUS examination. The median time between transplant and first EUS was 1537 days (range: 170-5204), whereas the median time between the two EUS examinations was 343 days (range: 304-648).

In all cases, EUS and enteroscopy were feasible and both examinations could be performed as scheduled within the same endoscopy session. In a vast majority of the patients, both procedures were indeed completed within $60 \mathrm{~min}$. No patient experienced severe discomfort or the need for premature termination of the procedures.

Patient 3 underwent graft enterectomy due to clinical and radiological suspicion of CR after her second EUS. CR was confirmed by the pathology examination of the explanted graft. The other patient with suspected CR (patient 5), died during follow-up due to COVID-19 infection. At that time, she suffered from severe malnutrition and strongly suspected CR supported by the findings at EUS+RI. No autopsy was performed.

\section{Endoscopic ultrasound}

In all cases, EUS was able to provide measurements of the thickness of both the entire intestinal wall and its different wall layers, figure 1 , table 2 . There were no obvious differences between the measurements taken during the first and the second EUS examinations.

Table 2 Overview of the endoscopic and microscopic findings

\begin{tabular}{|c|c|c|c|c|c|c|c|c|c|c|}
\hline Patient & & EUS 1 & & & EUS 2 & & $\mathrm{RI}$ & GITES & Histology & Clinical course \\
\hline & $1-V^{*}$ & I-III† & IV-V $\neq$ & $1-V^{*}$ & I-III† & IV-V $\neq$ & & & & \\
\hline 1 & 2.3 & 1.3 & 1.0 & 2.5 & 1.1 & 1.3 & 0.80 & Go & Normal & Uncomplicated \\
\hline 2 & 2.4 & 1.5 & 0.9 & 2.6 & 1.4 & 1.1 & $\mathrm{~N} / \mathrm{A}$ & Go & Normal & Uncomplicated \\
\hline \#3 & 2.5 & 1.2 & 1.3 & 2.8 & 1.4 & 1.4 & 0.93 & G1 & $\mathrm{CR}$ & $\mathrm{CR}$ \\
\hline 4 & 2.3 & 1.6 & 0.7 & 2.2 & 1.5 & 0.7 & $\mathrm{~N} / \mathrm{A}$ & Go & Normal & Uncomplicated \\
\hline 5 & 2.4 & 1.1 & 1.3 & 2.8 & 1.4 & 1.4 & 0.91 & G0-G1 & Suspected CR & $\mathrm{CR}$ \\
\hline 6 & 2.4 & 1.3 & 1.1 & 2.7 & 1.3 & 1.4 & 0.77 & Go & Normal & Uncomplicated \\
\hline 7 & 2.6 & 1.6 & 1.0 & 2.7 & 1.6 & 1.1 & 0.80 & Go & Normal & Uncomplicated \\
\hline 8 & 2.7 & 1.7 & 1.0 & 2.6 & 1.7 & 0.9 & 0.73 & Go & Normal & Uncomplicated \\
\hline 9 & 2.2 & 1.5 & 0.7 & - & - & - & 0.73 & Go & Normal & Uncomplicated \\
\hline 10 & 2.3 & 1.4 & 0.9 & - & - & - & 0.79 & Go & Normal & Uncomplicated \\
\hline 11 & 2.3 & 1.8 & 0.5 & - & - & - & 0.75 & Go & Normal & Uncomplicated \\
\hline
\end{tabular}

${ }^{*}$ Diameter of graft wall layer I-V (mucosa-serosa) in $\mathrm{mm}$.

†Diameter of graft wall layer I-III (mucosa-submucosa) in mm.

‡Diameter of graft wall layer IV-V (muscularis propria-serosa) in $\mathrm{mm}$.

CR, chronic rejection; GITES, Gothenburg Intestinal Transplant Endoscopy Score (G0-G4); RI, resistive index. 
The thickness measured of the entire wall (wall layer $\mathrm{I}-\mathrm{V}$ ) was comparable in the nine patients with an $\mathrm{AC}$ and in the two patients with suspected CR, table 2. In the two patients with suspected CR, the recorded thickness of wall layer IV-V (1.3 $\mathrm{mm}$ and $1.4 \mathrm{~mm}$, respectively) was numerically in the upper end of measurements performed in all 11 patients (range: $0.5-1.4 \mathrm{~mm}$ ), table 2 .

The EUS examinations allowed the identification and evaluation of the mesenteric vasculature, including the intramural vessels. In nine patients, we measured the $\mathrm{RI}$ in the mesenteric vessels adjacent to the intestinal wall, figure 2 . The RI was recorded $>0.9$ in both patients with suspected $\mathrm{CR}$, while the RI was recorded $\leq 0.8$ in all patients with an AC, table 2.

\section{ENTEROSCOPY}

The small bowel mucosa appeared normal (G0) at enteroscopy in all the nine patients with an uncomplicated clinical course, figure $3 \mathrm{~A}$ and table 2 . In the two patients with suspected CR, enteroscopy revealed blunted, dome-shaped villi, petechiae and a slightly friable mucosa (G1), figure $3 \mathrm{~B}$ and table 2 . No denuded areas were noted.

\section{HISTOPATHOLOGY}

In the nine patients with normal enteroscopy findings, microscopical examination of the mucosal biopsies revealed normal intestinal structure without signs of acute rejection and without positive staining for cytomegalovirus or C4d. In the two patients with clinical suspicion of CR, the microscopic examination of the mucosal biopsies uniformly found shorter villi as well as fibrosis and proliferating myofibroblasts in the lamina propria. In one case, besides mucosal atrophy and fibrosis, microscopy revealed low-grade acute rejection (increased apoptotic body count). The explanted intestinal graft (patient 3) showed mucosal atrophy as well as arteriosclerosis, fibrosis and scarring in the mesentery, all typical for CR. The assessment of the intestinal wall on the histological preparations from resected specimens was in line with findings recorded at EUS.

\section{DISCUSSION}

The present study is the first to report the use of EUS in the follow-up of the intestinal graft. The current findings suggest that EUS may assess the intestinal graft beyond the reach of endoscopic biopsies and provide a level of detail unavailable for other imaging methods used routinely such as MRI and CT.

Intestinal CR is an enteropathy with insidious, progressive course lacking early, specific clinical symptoms or mucosal findings at endoscopy. CR lacks reliable biomarkers in blood or faeces and the only indirect evidence may be only inferred by CT and MRI scans, showing mesenteric and intestinal wall thickening and a paucity of mesenteric vessels. ${ }^{5}$ Although most clinical series report an incidence of CR between $5 \%$ and $10 \%$, the largest single centre experience reported to date has identified CR as the most important single cause of graft loss $(30 \%)$, equaling infections and technical complications combined. ${ }^{17}$

The investigations summarised herein were initiated after a median interval of 5 years after ITx (first EUS) and they were repeated after about 1 year. Although the natural history of CR is poorly known, it is believed that its frequency increases 2 years after transplantation and reaches a peak during the third post-transplant year. ${ }^{18}$ Eight out of the 11 patients (all patients but patient 4, 8 and 10) in our study were within this time frame (time to second EUS counted) and, hence, were at risk of developing CR changes. In addition, the results of Nayyar et al infer that 1 year of observation may be sufficient to document ongoing CR making our yearly frequency of the examination relevant.

Endoscopic mucosal biopsies form the mainstay of intestinal allograft monitoring and allow the accurate diagnostic of acute rejection, infectious enteritis and posttransplant lymphoproliferative diseases. However, and importantly, the obstructive vasculopathy of the middlesized submucosal and mesenteric arteries, which are hallmark lesions of CR, cannot be visualised in endoscopic biopsies since they develop in the deeper wall layers or just outside the intestine. ${ }^{5}$ Our observations show that EUS is able to provide information about the intramural vascularity of the transplanted intestine and its adjacent mesentery. The method also allowed the measurement of an RI at the level of mesenteric vessels.

In spite of the limited number of observation, it seems that the mesenteric RI is increased in patients with clinical suspicion of CR. This finding is in line with the abundant experience from kidney and liver transplantation, where an increased RI $(>0.80)$ has been shown to be associated with allograft pathology. ${ }^{71819}$ The current field of research is relatively unexplored and, therefore, there is no established cut-off level for RI regarding intestinal grafts. Based on our data, it seems that the cut-off level for an increased RI might be higher in intestinal grafts (RI $>0.90$ ?). Why so? A possible explanation could be that the liver and the kidney are parenchymatous organs surrounded by a capsule, whereas the SI is a soft cavitary organ. We speculate that this relatively soft organ and its surrounding mesentery can reposition more freely within the abdomen, which hypothetically in turn might affect the vascular rheology and the RI. Future studies on the topic are required to validate, or question, the findings presented by us. The usefulness and significance of this newly described parameter, its interobserver variability as well as the circumstances that may influence it need to be established in larger patient series. Admittedly, measurement of the RI-index requires both adequate equipment and proper training of the endosonographer. Nevertheless, according to our experience, learning to master an RI measurement is relatively simple and not timeconsuming. The software of most modern ultrasound 
processors enables pulsed Doppler mode and thereby RI-measurement. Hence, neither of these two issues should be a limiting factor.

Graft dysmotility is a typical manifestation of CR and muscular alterations have been reported in explanted grafts and animal models. Experimental data indicate that severe damage to the muscularis and the enteric nervous system occurs before clinical or mucosal changes become apparent. ${ }^{20}$ Interestingly, intestinal smooth muscle hypertrophy and hyperplasia, associated with abnormal contractile and electrical activities, were observed in both rat and dog intestines with $\mathrm{CR} .{ }^{21}{ }^{22}$ An interesting and intriguing finding of the study is that total wall thickness did not appear obviously increased in the two patients with CR. On the other hand, our measurements were indicating a somewhat thicker outer part of the wall (the muscularis propria and the serosa) in the two patients with $\mathrm{CR}$ as compared with the nine patients with an AC.

In research, the clinical benefit of new techniques is always of interest. In the current study, we argue that EUS was an important factor in the overall evaluation of the graft function status facilitating decisions on clinical management. In both patients with CR (patient 3 and 5), EUS and RI were indicating abnormality of the graft pointing in the same direction as findings of other modalities.

Humbly, we admit that the number of patients included in the study was relatively small. Consequently, the results presented must be interpreted with prudence as findings in minor study populations are less valid by nature. Nevertheless, even from a limited sample size, one can obtain valuable indications on truly existing findings in larger study cohorts.

In order to facilitate the acute rejection surveillance in the early transplant period, all the examinations were performed through the ileostomy placed at the time of transplantation. Thereby, an easy and safe access to the transplanted small intestine was provided. We did not attempt to perform intestinal EUS through the transplanted or native colon after the ileostomy removal, as reaching the transplanted small bowel via the colon and by using the echoendoscope could be both technically challenging and risky. As an increasing proportion of ITx patients also receives a colon segment, ${ }^{4}$ further studies should explore the technical feasibility of this approach as CR seems to develop several years after transplantation, a time when the ileostomy may have already been removed in many patients (if constructed at all). ${ }^{23}$ The experience accumulated during the investigation of the upper gastrointestinal tract suggests that this may be a feasible alternative. ${ }^{24}$

Transabdominal ultrasound (TUS) with RI of the allograft could be an alternative approach in patients without an ileostomy. Besides the fact that TUS will gain access for measurements also in patients without an ileostomy, obviously TUS is less invasive than EUS. However, the drawback of TUS would be the risk of moderate quality ultrasound images both due to the larger distance from the echoprobe to the target organ and due to the risk of artefacts induced by reflecting intestinal gas. Nevertheless, we evaluated TUS as a complementary examination in four of the study patients. We recorded similar findings via the transabdominal approach, but the procedure was more time-consuming than EUS (approximately an additional $10 \mathrm{~min}$ as compared with EUS). The additional time was required for optimal positioning of the probe and the identification of appropriate bowel segments. Moreover, TUS would benefit from oral intake of macrogol solution by the patients some hours before TUS to minimise the volume of intestinal gas. Another aspect of the transabdominal approach is that endoscopy might be needed anyhow in order to visualise and assess the small bowel mucosa.

Routine, cross-sectional imaging (MRI) at predefined dates was not a part of the follow-up programme within the current study. Consequently, potential diagnostic information from this modality could not be recorded. Nonetheless, the resolution of MRI would not obviously be high enough for precise measurement and relevant comparison of the intestinal wall.

The current study has several limitations due to the low number of patients and observations as mentioned above. The external validity of the presented findings needs to be determined and multicentric, prospective studies would be welcome. The limited access to full wall biopsies will continue to remain a challenge for diagnosing CR and future related studies as these biopsies are almost impossible to obtain in this patient group except at ileostomy removal, at retransplantation or graft removal or at autopsy. ${ }^{56}$ We had access to full thickness biopsies in some of the patients, including in one case with clinical rejection (patient 3), allowing us to verify the EUS findings on actual samples obtained in a rather close time frame. In contrast, EUS may be repeated at any interval due to its minimal invasiveness and possibility to investigate rather extended segments of intestine as well as adjacent intestinal loops in the proximity of the segment bearing the ileostomy which is intubated.

In conclusion, the study provides a proof of concept on the use of EUS in the follow-up of the intestinal graft morphology and rheology in a group at risk of developing CR. The results show its technical feasibility and some previously unknown, relevant insights that warrant further expansion. EUS may provide useful information on the intestinal graft wall and the structures in its close proximity that may be used in the long-term monitoring and management of intestinal allograft recipients.

Acknowledgements All staff at the GEA Endoscopy Unit, Sahlgrenska University Hospital.

Contributors All authors planned and designed the study. All authors conducted the study. MO and PH analysed the data and performed the statistical calculations. $\mathrm{MO}, \mathrm{PH}$ and JV wrote the manuscript. All authors edited the final version of the manuscript. $\mathrm{MO}$ is the overall guarantor of the overall content in the article.

Funding Funding for this study was provided by grants from the Swedish state under the agreement between the Swedish government and the country councils (grants ALFGBG-695931, ALFGBG-812881, ALFGBG-875671, ALFGBG-875431, 
ALFGBG-716031, ALFGBG-50779 and ALFGBG-426801) and by grants from The Assar Gabrielsson Foundation (grant number: 17-20) and Magtarmfonden (grant number: 79211).

Competing interests None declared.

Patient consent for publication Not applicable.

Ethics approval This study involves human participants and was approved by the Regional Ethics Committee Board of West Sweden, Dnr 573-09. Participants gave informed consent to participate in the study before taking part.

Provenance and peer review Not commissioned; externally peer reviewed.

Data availability statement Data are available upon reasonable request.

Open access This is an open access article distributed in accordance with the Creative Commons Attribution Non Commercial (CC BY-NC 4.0) license, which permits others to distribute, remix, adapt, build upon this work non-commercially, and license their derivative works on different terms, provided the original work is properly cited, appropriate credit is given, any changes made indicated, and the use is non-commercial. See: http://creativecommons.org/licenses/by-nc/4.0/.

ORCID iD

Per Hedenström http://orcid.org/0000-0002-4106-1454

\section{REFERENCES}

1 Desai CS, Gruessner AC, Khan KM, et al. Isolated intestinal transplants vs. liver-intestinal transplants in adult patients in the United States: 22 yr of OPTN data. Clin Transplant 2012;26:622-8.

2 Varkey J, Simrén M, Jalanko H, et al. Fifteen years' experience of intestinal and multivisceral transplantation in the Nordic countries. Scand J Gastroenterol 2015;50:278-90.

3 Abu-Elmagd KM, Kosmach-Park B, Costa G, et al. Long-term survival, nutritional autonomy, and quality of life after intestinal and multivisceral transplantation. Ann Surg 2012;256:494-508.

4 Grant D, Abu-Elmagd K, Mazariegos G, et al. Intestinal transplant registry report: global activity and trends. Am J Transplant 2015;15:210-9.

5 Lauro A, Oltean M, Marino IR. Chronic rejection after intestinal transplant: where are we in order to AVERT it? Dig Dis Sci 2018;63:551-62.

6 Ruiz P. Updates on acute and chronic rejection in small bowel and multivisceral allografts. Curr Opin Organ Transplant 2014;19:293-302.

7 Stanescu AL, Hryhorczuk AL, Chang PT, et al. Pediatric abdominal organ transplantation: current indications, techniques, and imaging findings. Radiol Clin North Am 2016;54:281-302.

8 Bots S, Nylund K, Löwenberg M, et al. Ultrasound for assessing disease activity in IBD patients: a systematic review of activity scores. J Crohns Colitis 2018;12:920-9.
9 Coelho R, Ribeiro H, Maconi G. Bowel thickening in Crohn's disease: fibrosis or inflammation? diagnostic ultrasound imaging tools. Inflamm Bowel Dis 2017;23:23-34.

10 Kucharzik T, Wittig BM, Helwig U, et al. Use of intestinal ultrasound to monitor Crohn's disease activity. Clin Gastroenterol Hepatol 2017;15:535-42.

11 Crino SF, Rimbas M, Gabbrielli A, et al. Endoscopic ultrasound guided gallbladder interventions: a review of the current literature. $J$ Gastrointestin Liver Dis 2019;28:339-47.

12 Gondolesi GE, Fernandez A, Burghardt KM, et al. Meeting report of the XIV international small bowel transplant symposium: summary of presentations, workshops, and debates from a comprehensive meeting on intestinal failure, rehabilitation, and transplantation, Buenos Aires, Argentina, June 10-13, 2015. JPEN J Parenter Enteral Nutr 2018;42:477-89.

13 Hedenström P, Marschall H-U, Nilsson B, et al. High clinical impact and diagnostic accuracy of EUS-guided biopsy sampling of subepithelial lesions: a prospective, comparative study. Surg Endosc 2018;32:1304-13.

14 Naesens M, Heylen L, Lerut E, et al. Intrarenal resistive index after renal transplantation. N Engl J Med 2013;369:1797-806.

15 Varkey J, Stotzer P-O, Simrén M, et al. The endoscopic surveillance of the transplanted small intestine: a single center experience and a proposal for a grading score. Scand J Gastroenterol 2018;53:134-9.

16 Wu T, Abu-Elmagd K, Bond G, et al. A schema for histologic grading of small intestine allograft acute rejection. Transplantation 2003;75:1241-8.

17 Abu-Elmagd KM, Costa G, Bond GJ, et al. Five hundred intestina and multivisceral transplantations at a single center: major advances with new challenges. Ann Surg 2009;250:567-81.

18 Nayyar N, Mazariegos G, Ranganathan S, et al. Pediatric small bowel transplantation. Semin Pediatr Surg 2010;19:68-77.

19 Huang D-Z, Le G-R, Zhang Q-P, et al. The value of color Doppler ultrasonography in monitoring normal orthotopic liver transplantation and postoperative complications. Hepatobiliary Pancreat Dis Int 2003;2:54-8.

20 Uzochukwu LN, Bluth El, Smetherman DH, et al. Early postoperative hepatic sonography as a predictor of vascular and biliary complications in adult orthotopic liver transplant patients. AJR Am J Roentgenol 2005;185:1558-70.

21 Heeckt PF, Halfter WM, Schraut WH, et al. Small bowel transplantation and chronic rejection alter rat intestinal smooth muscle structure and function. Surgery 1993;114:449-56.

22 Sugitani A, Bauer AJ, Reynolds JC, et al. The effect of small bowe transplantation on the morphology and physiology of intestinal muscle: a comparison of autografts versus allografts in dogs. Transplantation 1997:63:186-94.

23 Moon JI, Zhang H, Waldron L, et al. "Stoma or no stoma": First report of intestinal transplantation without stoma. Am J Transplant 2020;20:3550-7.

24 Katanuma A, Hayashi T, Kin T, et al. Interventional endoscopic ultrasonography in patients with surgically altered anatomy: techniques and literature review. Dig Endosc 2020;32:263-74. 